\title{
Systemic functional adaptedness and domain-general cognition: broadening the scope of evolutionary psychology
}

Michael Lundie, The University of Texas at Dallas

\begin{abstract}
Evolutionary Psychology tends to be associated with a massively modular cognitive architecture. On this framework of human cognition, an assembly of specialized information processors called modules developed under selection pressures encountered throughout the phylogenic history of hominids. Accordingly, the coordinated activity of domain-specific modules carries out all the processes of belief fixation, abstract reasoning, and other facets of central cognition. Against the massive modularity thesis, I defend an account of systemic functional adaptedness, which holds that non-modular systems emerged because of adaptive problems imposed by the intrinsic physiology of the evolving human brain. The proposed reformulation of evolutionary theorizing draws from neural network models and Cummins' (1975) account of systemic functions to identify selection pressures that gave rise to nonmodular, domain-general mechanisms in cognitive architecture.
\end{abstract}

Keywords: Adaptation; Connectome; Modularity; Rich Club; Systemic Function; Selection Pressure

Acknowledgements: I am grateful to Daniel Weiskopf, Neil Van Leeuwen, Andrea Scarantino, David Washburn, Daniel Krawczyk, and Matthias Michel for valuable feedback on previous drafts. Much appreciation also goes to the anonymous reviewers for their constructive commentary. The points raised in their assessments significantly benefitted the revised manuscript.

Address for Correspondence: School of Behavioral and Brain Sciences, The University of Texas at Dallas, Richardson, TX, USA 75080

Email: Michael.Lundie@utdallas.edu 


\section{Introduction}

There are sundry examples of specialized solutions to adaptive problems documented throughout biology (Sperber 1994; Carruthers 2006). Consider echolocation in bats, a specialization that enables predation of insect prey; color change in octopuses, to better evade detection by roaming predators; garish color displays in birds, to attract viable mates. Such specialized organ systems emerge in large part from a particular selection pressure (Boyer 2015: 186; Buss 1995: 2; Cosmides and Tooby 1994: 86). ${ }^{1}$ Specialized adaptations carry out wellcircumscribed functions to secure an organism's survival and reproduction (Godfrey-Smith 2013: 51). Scaling up to a staggeringly complex organ system such as the human brain, a veritable Swiss-army knife in the scope of its functional repertoire, there appears to be an exception to this rule of specialization (Mithen 1996). The brain's computational systems must carry out perceptual processes like vision and audition in addition to higher-order processes in central cognition that mediate reasoning, belief formation, and other facets of distinctively human thought. With such a sweeping range of functions, there appears to be no specific adaptive problem for which the brain's cognitive architecture is adapted or specialized. At first approximation, the brain appears to be a general domain learning and computation system.

\footnotetext{
${ }^{1}$ The strength of this claim notwithstanding, cases of multifunctional mechanisms and traits arising by means other than natural selection have been documented since Gould and Vrba (1982: 6). Take, for instance, the category of exaptations, which are adaptations that are coopted to serve additional functions, as well as spandrels that emerged as developmental byproducts of adaptations.
} 
And yet, in recent decades, some cognitive scientists have come to regard the brain's seeming domain-generality as illusory (see Carruthers 2006; Tooby and Cosmides 2005; Sperber 2004). Beneath this facade of domain-generality lies an ensemble of specialized neural systems. The research program of evolutionary psychology has offered a method of analysis to identify the functional characteristics of these specialized neural systems. Evolutionary psychologists have impacted cognitive science by identifying sets of selection pressures that spurred the evolutionary development of generalizable (species-specific) neural structures that carry out cognitive operations (Fawcett et al. 2014; Sober 1984: 211). Befitting the Swiss-army knife metaphor, the cognitive mind is envisioned as a collection of specialized cognitive modules (Sperber 2004: 53-4), a theoretical orientation that has led a number of evolutionary psychologists to argue that the cognitive mind is massively modular (MM) - that is, exhaustively or mostly constituted by specialized modules (see Carruthers 2006; Sperber 2002; 2004).

My aim is to challenge the MM thesis and make the case for a domain-general cognitive architecture. To start, section 1 sketches the theoretical link from evolutionary psychology to MM cognitive architectures by establishing how Cosmides and Tooby’s (1997) selection pressures argument motivates the MM thesis. Section 2 lays out a rebuttal to the selection pressures argument. It is here where I propose an account of systemic functional adaptedness, drawing on findings from network theory and Cummins' (1975) account of systemic functions to reveal how adaptive problems imposed by the physiology of the evolving human brain created a selection pressure for non-modular structures in the cognitive mind. Anticipating counterarguments, section 3 explores potential objections on behalf of the MM thesis, followed in turn by responses to those objections in section 4 . I conclude by exploring directions for further 
developing a domain-general cognitive architecture based on a broadened understanding of evolutionary theorizing.

\section{From Evolutionary Psychology to Massive Modularity}

The evolutionary psychologists David Buss (1995) and Tooby and Cosmides (1994; 1995; 1997) spurred the development of evolutionary psychology, a field subsuming and integrating the disparate psychological theories of the human mind under a unified set of paradigmatic principles. These paradigmatic principles include fitness, adaptation, and selection pressure ${ }^{2}$ all conceptual elements that capture an ecological model called the environment of evolutionary adaptedness (Boyer 2015: 189; Buss 2005; Tooby and Cosmides 1987: 5, 1994: 87). The environment of evolutionary adaptedness most relevant to forming hypotheses about cognition reaches back to the Pleistocene era (Buller 2005: 9; Tooby and Cosmides 1994: 87). During this period, prehistoric hunter-gatherers struggled to overcome a host of adaptive problems relating to resource acquisition, avoiding dangerous predators, outmaneuvering conspecific rivals, securing shelter, finding mates, and raising offspring (Buss 1995: 9-10). Hominid variants lacking such capacities were less fit, and therefore were less likely to reproduce, resulting in the propagation of fitness-enhancing traits in subsequent generations (Buss 1995). Fitness is a measurement of an organism's capacity to overcome adaptive problems, a function of survival and reproduction which enables the organism to pass along genes encoding for those adaptive traits to the next

\footnotetext{
2 Additional concepts relevant to evolutionary psychology are "regulation," "computational architecture," "organization," "design," "entropy," "replication," "by-product," and "task environment" (see Cosmides and Tooby [1987] for an overview).
} 
generation. Some adaptive traits encode for features of the cognitive mind, leading to the development of "mental organs" equipped with inference rules suited to overcoming various adaptive problems (Pinker 1997: 21). The term "module" captures a more refined conceptual understanding of these specialized mental organs.

\subsection{The Three Characteristics of Cognitive Modules}

At the most general level, modules correspond to specialized computational mechanisms ${ }^{3}$ that carry out cognitive functions (Fodor 1983: 36-38; 2000: 91). There is not much consensus on the necessary and sufficient conditions that define modularity (Carruthers 2006: 3). However, in order to evaluate arguments in favor of massive modularity, we must settle on a minimally tendentious construal of modularity (but see Zirilli [2016] for a defense of so-called 'softly' defined modules - a minimalist construal eschewing strict definitions of modularity which this paper does not address). In The Modularity of Mind, Fodor proposed nine distinct features that characterize modules (1983: 47-101). ${ }^{4}$ It will suffice for present purposes to regard modules as distinguished by the following three properties: (1) domain-specificity, (2) encapsulation, and (3) mandatory operation (Fodor 1983: 36-7, 47, 52, 64; and see Carruthers [2006] and Sperber [2004] for further elaboration on characteristics of modularity).

\footnotetext{
${ }^{3}$ Describing modules as computational systems equivocates between two senses of computation (Samuels 1998: 579). Modules may carry out computations under either the hardware conception or the algorithm conception (Jungé and Dennett 2010). In the hardware sense, modules are localized in specific brain regions. On the latter interpretation, modules as specialized sub-routines or mental programs - on this account modules could be implemented across discontinuous neural regions (see Samuels 1998: 579). The massive modularity thesis critiqued in this paper refers to the more mainstream algorithmic construal of modularity posited by Carruthers (2006) and Sperber (2004).

${ }^{4}$ According to Fodor's original formulation (1983), modules are: (1) localized, (2) subject to characteristic breakdowns, (3) mandatory, (4) fast, (5) shallow, (6) ontogenetically determined, (7) domain specific, (8) inaccessible, and (9) informationally encapsulated.
} 
Starting with (1), modules are domain-specific insofar as they process a specific type of input or deliver a specific output to perform a cognitive function (Carruthers 2006; Cosmides and Tooby 1994: 94). Modules governing perception relay sensory information about the environment to relevant cortical regions that process these input data. Modules in higher-order processing regions perform further computations. These computations manipulate outputs as components in central cognitive operations such as reasoning and decision making.

Modules are also (2) encapsulated in the sense of being computationally impenetrable by other modules and have access to only their own proprietary databases. Put succinctly, the informational databases of modular systems are dissociable and opaque to one another (Weiskopf 2010: 8). By regarding modules as dissociable computational systems, the flow of information in cognition is restricted only to modules whose informational domains are sufficiently relevant to current task demands (Sperber 2004: 60-1).

There is less convergence on whether (3) mandatory operations is definitive of modularity, but I include it because a number of MM architectural frameworks formulate modularity accordingly (see, e.g., Sperber 2004: 60). Akin to a 'cognitive reflex,' modules operate mandatorily in the sense of automatically processing appropriate perceptual inputs (i.e., appropriate in the sense of satisfying a module's activation conditions). Once initiated, modular procedures cannot be consciously blocked (Sperber 2004: 60-1). Optical illusions usefully illustrate mandatory operation. Consider, for instance, the persistence of the Müller-Lyer illusion - where two lines of equal length appear to be of different lengths. Even as the observer recognizes the illusion, she cannot consciously block the illusion from manifesting. This effect demonstrates how perceptual modules mandatorily perform the operations that generate the 
illusion (see Zeman et al. [2013] for an explanation of the computational mechanisms underlying the Müller-Lyer illusion).

\subsection{Reverse Engineering the Massively Modular Mind}

Having covered the three basic characteristics of modularity, we can follow the inference from the reverse-engineering methodology of evolutionary psychology to the massive modularity thesis (abbreviated as "MM" from this point on). If cognitive modules gradually arose throughout phylogenic history, then it should be possible to reverse engineer modular functions in terms of the relevant selection pressures and adaptations found in the ancestral Environment of Evolutionary Adaptedness (Cosmides and Tooby 1997). Conversely, if every cognitive module emerged as an adaptation to selection pressures, then the cognitive mind is constituted by modules that collectively facilitate central cognition (Carruthers 2013a: 8; Sperber 2004: 54).

In summary, the unifying thesis of MM is to regard our cognitive architecture as composed of an assembly of modules all working in concert to mediate cognitive operations. Some theorists (Cosmides and Tooby 1992; Sperber 2004; Carruthers 2006) hold the strong view that most features of perception and central cognition are governed by domain-specific modules. ${ }^{5}$ The figure below illustrates the strong thesis of MM:

\footnotetext{
${ }^{5}$ Some modularists, including Carruthers (2013a, 2013b), as well as Cosmides and Tooby (2000), regard the strong MM thesis as compatible with there being some mechanisms, such as working memory, that exhibit domain-general functionality. Nevertheless, the overarching framework of MM maintains that central cognition is predominantly constituted by domain-specific modules (Cosmides and Tooby 2000: 1171, 1261).
} 


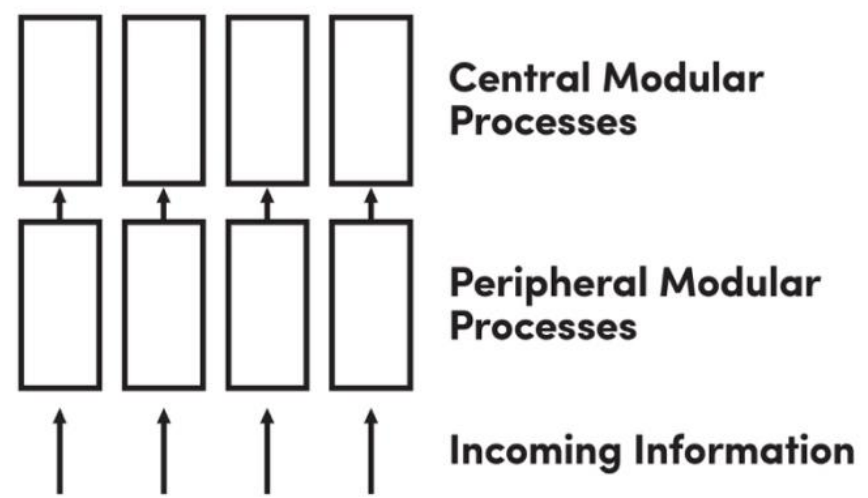

Fig. 1: Theoretical Schema of Massive Modularity

Diagramed above is a simplified model of the order of information processing within the MM cognitive architecture. What makes this model massively modular is ascribing modular systems to perception (the peripheral processes) as well as central cognition (cf. Sperber [2004], and Carruthers [2006]). From Nettle (2007: 261), modified.

Fig. 1 illustrates how, first, peripheral modules process incoming perceptual information to identify objects and properties of the external environment. Outputs from the peripheral layer are then relayed further downstream to central modular processes that carry out tasks relevant to higher-order reasoning, belief evaluation, and decision making (Carruthers 2013: 143). ${ }^{6}$ Proponents of MM therefore draw on the reverse-engineering of evolutionary psychology to systematically identify the selection pressures that spurred the development of modules in cognitive architecture.

How, then, according to the MM theory did it come about that a cognitive architecture adapted to ancestral environments could be capable of interfacing with the modern world? Artificial environments of the present day radically differ from the African veldt traversed by hunter-gatherer ancestors (e.g., there were no cell phones and automobiles in the Pleistocene, so

\footnotetext{
${ }^{6} \mathrm{MM}$ theorists differ on the assignment of roles to the modules that govern central cognition. According to Carruthers's (2006) MM framework the language content-integrator is a higher-order module that performs complex cognitive operations, whereas on Sperber's $(1994,2000)$ account the metarepresentation module plays a similar role.
} 
how do modern minds master tools that lack prehistoric analogs?). Sperber (2004) has proposed an explanation. The proposal is that activation conditions of a cognitive module may fall within the activation conditions of either the module's proper domain or actual domain (Sperber 2004: 55). The module's proper domain refers to the input parameters and functional properties for which it was selected (Sperber 2004: 55; Buller 2005: 57). Take, for instance, the facerecognition system. The adaptive benefit of evolving a module that identifies different faces relates to the importance of tracking conspecific rivals and potential mates, distinguishing friend from foe, kin from non-kin, etc. These adaptive problems created a selection pressure for a facerecognition module that was retained as a universal feature of human cognition due to its adaptive benefit (Tooby and Cosmides 1987: 42). Dedicated neural regions residing primarily in the Fusiform Face Area (FFA) gradually evolved to carry out computations inherent to the module (Green 2016). Accordingly, the face-recognition module's proper domain corresponds to the perceptual cues exhibited by human faces. However, structural properties sufficiently similar to that of a human face may activate the face-recognition system, which refers to the facerecognition module's actual domain (Sperber 2004: 55). This expanded range of inputs allows for the perception of 'faces' in abstract works of modern art, or in a jagged rock formation on the mountainside. One corollary is that inputs falling outside the module's actual domain will not activate it. This theoretical adjustment explains how modern minds navigate through artificial environments by responding to inputs that fall within the actual domain of cognitive modules.

Another potential complication is to account for the flexibility of central cognitive processes in the human mind. How does our cognitive architecture combine concepts to compose novel and complex mental representations? What mechanisms enable this compositionality of thought (see Fodor and Lepore 1996)? Such capacities would support, for example, comprehension of 
metaphor (Nettle 2007) and the integration of relations and semantic contents in analogical reasoning (Bunge 2005; Krawczyk 2010). To account for these capacities, some theorists have posited a domain-general central system in cognitive architecture (see Fodor 1983; Elman et al. 1996; Karmiloff-Smith 1992; Prinz 2006; Quartz and Sejnowski 1997; Samuels 1998;

Woodward and Cowie 2004). A domain-general system allows for the peculiar "inferential promiscuousness" of the cognitive mind (Evans 1982). This property refers to the mind's capacity to combine any token proposition with any other token proposition and iteratively carry out further inferences (Brewer 1999; Hurley 2006). For example, a Fodorian (1983) cognitive architecture posits a central system that carries out procedures in belief fixation, abstract and abductive reasoning, and other capacities that reflect inferential promiscuousness. This approach stands in contrast to the MM thesis because it incorporates non-modular mechanisms that mediate central cognition. See fig. 2 below for a simplified theoretical schema of non-modular cognitive architectures:

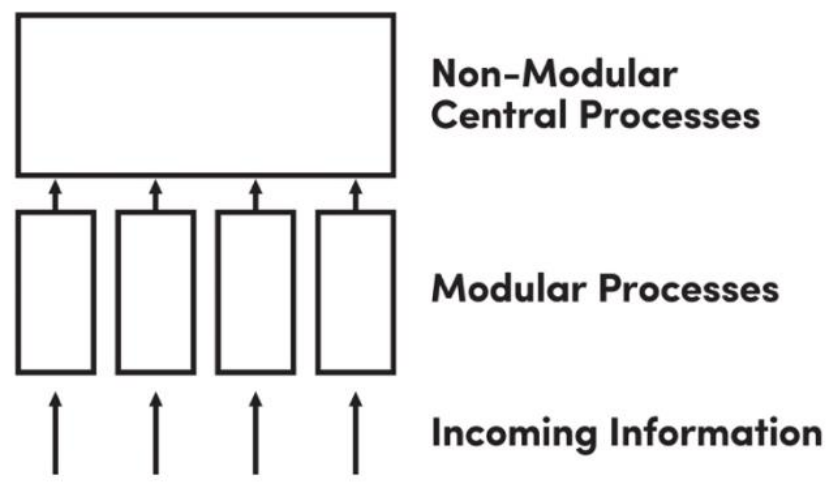


Fig. 2: Theoretical Schema of Domain General, Non-Massively Modular Cognition

Diagramed above is a simplified schema of the order of information processing within the non-MM cognitive architectures. Note that peripheral systems like perception may be governed by mechanisms that satisfy the conditions for modularity. So the alternative to massively modular architectures may concede that some mechanisms are modular, while reserving central cognition for non-modular mechanisms (cf. Fodor 1983, 2000). From Nettle (2007: 261), modified.

To understand the non-modular central system, let us turn to a description of domain-general cognition. Buller (2006: 151-2) describes domain-general cognition as following generally applicable rules of logic and content-free algorithms to form beliefs and inferences across an unrestricted range of input domains (see Fodor 2000: 60-2, for further elaboration on domaingeneral cognition). An example of one such logical rule is modus ponens: (1) $P \rightarrow Q$, (2) $P$, therefore (3) $Q$. For example, $S$ uses modus ponens to deduce (1) if the sun shines, my plants will grow, (2) the sun is shining, $T f(3)$ my plants will grow. Likewise, $S$ can also reason (1) If the sun's light does not reach the plants, then there must be an obstruction blocking the sunlight (2) the sun's light is not reaching the plants, Tf (3) there must be an obstruction blocking the sunlight. A domain-general central system systematically and recursively generates beliefs using formal procedures of reasoning like modus ponens (Fodor 1994).

Domain-general central systems would also be unencapsulated and flexible. In order to operate according to domain-general rules like modus ponens, the relevant mechanisms should be unencapsulated in their capacity to recruit from multitudes of cognitive databases (Weiskopf 2014:17). Retrieval and association of mental contents, particularly in analogical reasoning, may traverse the divisions that separate semantic domains (Krawczyk 2018; Holyoak 2012). For example, in order to perform analogical reasoning $S$ may initiate inferences that incorporate semantic knowledge within the domain of botany (e.g., to identify the optimal growth conditions plant species $x$ match those of a similar plant species $y$ ), or even draw upon outside-domain 
knowledge in finance to understand how photosynthesis works (e.g., to recognize that solar energy inducing the storage of glucose in plants is similar to making monetary investments in a savings account). Moreover, an unencapsulated central systems would be flexible so as to engender the functional capacity to switch tasks, recursively generate chains of inferences, and revise beliefs in light of contrary information (Fodor 2000). For example, suppose $S$ discovers that her plants are not growing. The next step would be to figure out whether there is an obstruction blocking the sunlight, or whether the plants are not getting enough water. Such flexibility enables the iteration of complex sequences of reasoning procedures, thereby initiating and terminating chains of inference at will.

\subsection{The Selection Pressures Argument Against Domain-General Central Systems}

We turn now to the selection pressures argument against the tenability of using evolutionary theorizing to account for domain-general central systems. Cosmides and Tooby (1997) have formulated the hypothesis that highly specific selection pressures in the Environment of Evolutionary Adaptedness, such as resource gathering and predator detection, led to the development of domain-specific modules. Inherent to this reverse engineering methodology is a theoretical orientation toward externalism, which asserts that adaptive problems that created selection pressures are found in features of the ancestral environment - e.g. resources and predators (Cosmides and Tooby 1997: 81). Conversely, each selection pressure found in the external environment corresponds to domain-specific solution. Therefore, the cognitive mind could not be governed by a general-purpose learning system or all-purpose problem solver. Cosmides and Tooby (1994) clarify, “domain-specific cognitive mechanisms, with design features that exploit the stable structural features of evolutionarily recurring situations, can be 
expected to systematically outperform (and hence preclude or replace) more general mechanisms that fail to exploit these features" (90, emphasis added).

For domain-general features in cognition to have evolved, hunter gatherer ancestors must have encountered a corresponding set of adaptive problems. However, as Symons (1992) put it, "There is no such thing as a 'general problem solver' because there is no such thing as a general problem" (142, emphasis added). Selection pressures correspond to stable statistical regularities in the environment (e.g. clumped resources, cues of predatory threat) (Tooby and Cosmides 1987). Recurrent statistical features in the environment are fine-grained enough to select for specialized adaptive structures like modules (Tooby and Cosmides 1987: 53, footnote). One such statistical regularity would be animate objects that could correspond to dangerous predators. This regularity would select for animacy-detection systems that assist in the detection of such threats (Caramazza and Shelton 1998). Cosmides and Tooby (1992: 113, 1995: xiii) assert that nearly all the major facets of central cognition can be readily accounted for by modules specialized for spatial relations, tool-use, social-exchange, kin-oriented motivation, semantic inference, communication pragmatics, theory-of-mind, and so on.

Lending further support, Sperber (2004) observes that even a seeming domain-general logical rule like modus ponens could be governed by a dedicated module (2004: 56 , footnote). Modus ponens is constrained by strictly defined input conditions. Appropriate inputs are pairs of premises that conform to the syntactical structure of modus ponens but need not draw on the actual propositional or semantic content of those premises. Sperber (2004) elaborates, $[\ldots]$ The difference between a wholly general and the number-specific modus ponens is one of inputs, and therefore of domain-specificity, not one of database, and therefore not of encapsulation [...] In particular, they ignore data that might cause a rational agent to 
refrain from performing the modus ponens and to question one or other of the premises instead (Harman 1986). If there is a modus ponens inference procedure in the human mind, it is better viewed, I would argue, as a cognitive reflex. (56, footnote, my emphasis)

Therefore, even a putatively domain-general process like modus ponens inferences could be (1) domain-specific, (2) encapsulated, and (3) mandatory in operation. And if each module in central cognition corresponds to external selection pressures found in the Environment of Evolutionary Adaptedness, then it remains unclear how non-modular cognitive mechanisms could have evolved.

The theoretical basis of the selection pressures argument is found in the individuation of cognitive mechanisms by reference to adaptive problems found in the Environment of Evolutionary Adaptedness. The implication is that adaptive problems driving the evolutionary development of cognition were instantiated in statistical features of the ancestral environment. Examples include cooperative and rivalrous interaction with conspecifics, acquisition of resources, and avoiding predation. In their framing of the argument for the MM thesis, Cosmides and Tooby (2005) would contend that selection pressures exogenous to the organism account for the most significant aspects of cognitive architecture (see Godfrey-Smith [1996: 30-65] for detailed discussion of the reverse-engineering methodology forming the basis of the selection pressures argument).

\section{Systemic Functional Adaptedness and Cognitive Architecture}

In this section, I develop a rebuttal to the selection pressures argument. Note that I do not aim to discount altogether the research program of evolutionary psychology. Although it bears 
acknowledgment upfront that there is an extensive critical literature on the reverse-engineering methodology of evolutionary psychology (for insightful critical analysis of evolutionary psychology, see Davies, Fetzer and Foster [1995], Woodward and Cowie [2004], and Buller [2005]). However, this critical literature falls outside the scope of this paper, for the present aim is to propose a methodological retooling of evolutionary psychology to widen its explanatory scope. The crux of the dispute as I frame it here concerns how cognitive systems are individuated by the brand of evolutionary psychology represented by Cosmides and Tooby (sometimes referred to as the "Santa Barbara" approach, abbreviated as "EP" to highlight its distinctive theoretical commitment, including its association with the MM thesis). The EP approach developed by Cosmides and Tooby (2005) individuates mechanisms by reference to external selection pressures, whereas the broadened paradigm I propose here rejects the MM thesis and instead analyzes cognitive systems by reference to physiological factors of the containing neural system. What unfolds is an exposition of the evolutionary processes that would favor the emergence of non-modular and domain general properties of cognitive architecture.

What EP neglects is the range of adaptive problems that do not occur in the Environment of Evolutionary Adaptedness, but are found instead in endogenous properties of the evolving human brain. ${ }^{7}$ It was observed by Rosch (1978: 3) that a viable neural architecture is constrained by a general principle of cognitive economy, referring to the mandate of optimizing distribution of information in a neural system while conserving finite metabolic resources. The brain is replete with sub-systems designed to carry out various functions (e.g., cortical areas dedicated to memory, vision and audition, and language processing). But each additional neural component

\footnotetext{
${ }^{7}$ In his review of What Darwin Got Wrong, Godfrey-Smith (2010) points out how Fodor and Piattelli-Palmarini (2010) describe factors of the internal structure of the organism playing a role in determining which adaptations emerge.
} 
incurs a metabolic cost. Moreover, the accretion of different sub-systems potentially disrupts the established neural structure and its pre-existing functional capacities (Bullmore and Sporns 2012: 336). The accretion of additional sub-systems would have occurred in tandem with expanding cortical volume; one could infer a descent-with-modification selection process favoring compensatory mechanisms that would offset metabolic costs while maintaining pre-existing functions (Barrett 2012). The selection process would generate mechanisms that structurally and functionally integrate the more recently evolved cognitive systems. Structural and functional integration of multiple sub-systems is prerequisite to the performance of complex computations inherent to central cognition (Sporns and Bullmore 2010; 2012: 336).

Neural network theory and graph theory supply useful models for illustrating how factors of the cognitive economy shaped the organization of the connectome (Bullmore and Sporns 2009). De Reus and van den Heuvel (2014) define the connectome as "the complex network of all neural elements and neural connections of an organism that provides the anatomical foundations for emerging dynamic functions." Bullmore and Sporns' (2012) network model shows how some variants of connectome organization, each defined over different neural network topologies, are more metabolically efficient than other variants. Their analysis also uncovered the adaptive challenges that created a demand for a narrow range of network topologies (2012: 338). The first was minimizing metabolic inefficiencies, measured as a function of wiring structurally distinct neural regions; the second was maximizing the distribution of neural information to functionally integrate systems related to central cognition. The interplay of these two factors of structural and functional integration (interchangeably called 'connectivity') favored variants in connectome organization that struck a balance between the two physiological demands (Sporns 2013; Sporns 2012: 347; Sporns 2011: 134-9). 
Consider further the fitness value conferred by structural and functional connectivity (Bullmore and Sporns 2009). In the hypothesis space of permutations of connectome organization there are measurable deviations in fitness-value along the two dimensions of functional capacity and metabolic efficiency (Chklovskii and Koulakov 2004; Kaiser and Hilgetag 2006; van den Heuvel et al. 2012; Sporns 2013; Sporns 2011). Over-segregation of neural processing systems without structural integration can impede functional performance in central cognition (Bullmore and Sporns 2012). De Reus and van den Heuvel (2014: 2) clarify that, without sufficient structural interconnectivity, the global exchange of neural information among distinct processing systems would be compromised. For example, neural systems that are unsuitably structured would impede metabolic efficiency and disrupt the coordinated activity of cognitive systems (Sporns 2011: 127-8). Therefore, maladaptive structural arrangements of cognitive mechanisms within the organism, no less than external adaptive problems concerning mate selection or resource acquisition, may present a host of potential impediments to survival and reproduction (Sterelny and Griffiths 1999: 352).

Maladaptive neural organization and metabolic inefficiencies could have impeded the evolutionary development of additional cognitive functions from evolving, especially on the relatively short time-scale on which rapid neocortical magnification took place (Sporns 2011; Chklovskii and Koulakov 2004; Kaiser and Hilgetag 2006). Such adaptive challenges would have impeded the evolution of cognitive functions that require complex informational integration and coordinated activity of neural structures (Godfrey-Smith 2013: 53). The computational demands imposed by reasoning and abstract thought would have required the structural and functional integration of relevant cognitive systems during the evolution of the brain. Thus, 
parameters defined by the cognitive economy selected for a narrow range of neural wiring variants that are prerequisite to the evolution of central cognition.

The fitness value of network properties that maintain the delicate homeostatic balance between functional integration and metabolic efficiency may be denoted as systemic functional adaptedness. Reverse engineering the structural and functional properties of the connectome enables the inference of such mechanisms that mitigate metabolic cost and facilitate distribution of neural information in higher-order cognitive functions (Sporns and Bullmore 2010; 2012: 343). Studies of the particular topological arrangement in the human connectome have uncovered such fitness-enhancing properties. For instance, a network analysis conducted by Liang et al. (2017) revealed neural network components that minimize metabolic cost while maintaining functional connectivity in the brain.

To understand how network structures may engender systemic functional adaptedness, it is helpful to invoke Cummins' (1975) theory of systemic functions. To define the functional repertoire of a cognitive mechanism, we must identify the causal contributions made by a mechanism relative to the functions of the containing system. More specifically, a mechanism is individuated by analyzing the structural and functional benefits it imparts to the encompassing neural system. Some philosophers have argued for a systemic construal of functions at the exclusion of selected functions (see Amundson and Lauder 1994). The account I defend, however, follows Davies (2000) by regarding systemic functions and selected functions as compatible categories by which to individuate cognitive structures. By viewing systemic functions through evolutionary lenses, some components in the connectome may be individuated by their causal role in effectively maintaining the optimal balance of informational distribution and metabolic efficiency in the connectome. According to this view, traits encoding for these 
components were retained in evolutionary history due to the fitness benefits imparted by systemic functions.

There is ample evidence from connectomics suggesting that a centrally located network structure called the "rich club" facilitates a host of systemic functions (Bullmore and Sporns 2012: 342; Sporns 2013; van den Heuvel and Sporns 2011). The rich club is a point of convergence in the connectome that boasts the highest degree of dense interconnectivity among neural hubs (van de Heuvel et al. 2012). One causal function of the rich club is to support functional coupling among sub-system across the connectome (Bullmore and Sporns 2012: 343).

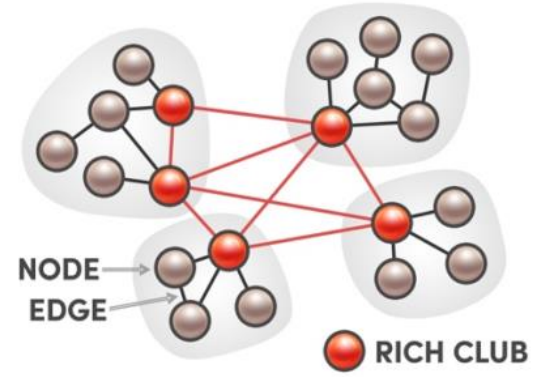

Fig. 3: Rich Clubs in the Connectome

Densely interconnected regions within the connectome correspond to rich clubs that assist in efficient information flow. Distal connections in the rich club are metabolically expensive, suggesting an important functional and integrative role to offset the metabolic investment costs by the organism. From Box 3, "Communities, cores, and rich clubs" (Bullmore and Sporns 2012: 342), modified.

Nested within the rich club is a more centralized structure called the "hub core" which creates further linkages across connector hubs (Sporns and Bullmore 2010; 2012: 342). Among the hub core's systemic functions is to support information flow across the topologically distant nodes in the connectome. 


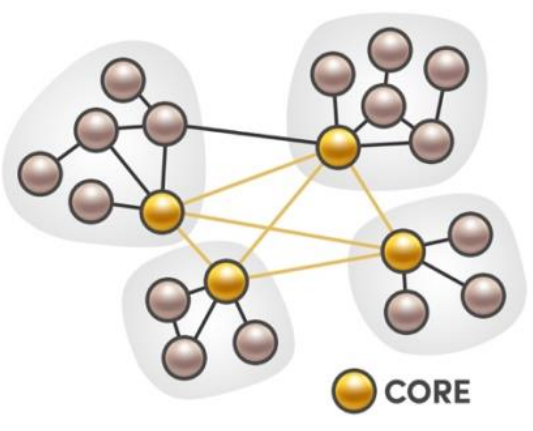

Fig. 4: Hub Core in the Connectome

Inter-modular connector hubs occupy a topologically more central or potential 'bottleneck' role between subsystems. An integrated core of densely inter-connected hubs has a central role in generating globally efficient information flow and integration. From Box 3, "Communities, cores, and rich clubs" (Bullmore and Sporns 2012: 342), modified.

With a suitable formulation of systemic functional adaptedness in hand, how do the rich club and hub core structures implement systemic functions, and how could their intrinsic functionality map onto a domain-general cognitive architecture?

Essential features of central cognition are facilitated by integrative functions that, according to whole-brain computational modeling, are coordinated by the rich club and hub core (Senden et al. 2017). An integrative system that combines, compares, and evaluates information is what engenders central cognition with its distinctive operations of flexible task-setting, goal valuation, and reasoning (for detailed analysis of the functional correlates of central systems, see Boureau, Sokol-Hessner, and Daw, [2015]). Centralized network structures participate in a range of discrete resting state networks (RSNs), including the fronto-parietal control network, whose components support the deployment and maintenance of task-oriented attention and executive control, and these centralized network structures also appear in the default mode network, which supports simulations of future events and reflection on knowledge about one's self and others (Unsworth and Robison 2017; Grayson et al. 2014; van den Heuvel and Sporns 2013; Vincent et 
al. 2008). Cortical components, especially the anterior insular cortex, of another RSN called the salience network support selective attention of task-relevant information for entry into central cognition (Michel 2017; Uddin 2015). These RSNs draw from specialized neural sub-systems represented in the connectome as modular communities of nodes and their local connections (Bullmore and Sporns 2012; van den Heuvel et al. 2012).

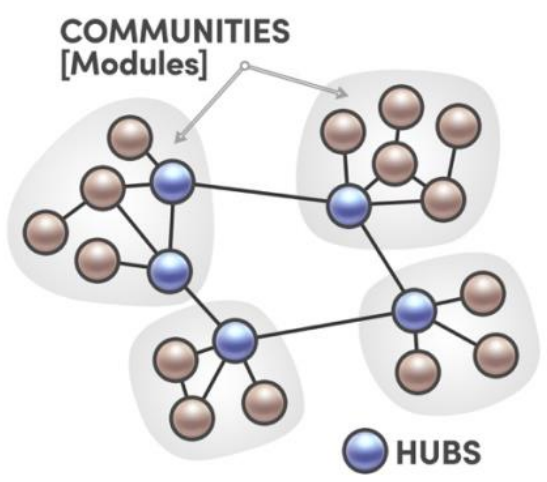

Fig. 5: Communities (Modules) in the Connectome

Communities connected by hubs form specialized neural communities. Density of connections is generally greater within a community than between communities. Computational studies highlight the advantages of specialized organization: modular networks deal more effectively with the increased processing demands imposed by variable environments; additionally, modularity confers a degree of resilience against dynamic perturbations and small variations in structural connectivity. From Box 3, "Communities, cores, and rich clubs" (Bullmore and Sporns 2012: 342), modified.

Not to be confused with the cognitive modules defined in section 1 , modules in the parlance of network and graph theory are typically understood as localizable neural communities that carry a more restricted range of circumscribed functions in contrast to the network correlates of central cognition. The cortical correlates of network modules encompass "occipital and parietal visual and sensory regions, temporal auditory regions, frontal (pre)motor regions, as well as insular, medioparietal, and mediofrontal regions overlapping the limbic system" (de Reus and van den Heuvel 2013). Note that the cognitive architecture proposed supported by these models 
are noncommittal on the question of whether network communities (or some sub-set thereof) may actually satisfy the three conditions that define cognitive modules.

Crucially, the capacity to functionally integrate network communities is a distinctive systemic function of the mechanisms that carry out central cognition. Increasingly interconnected neural networks and magnified cortical volume translated to advances in the computational power of the evolving human brain (Herculano-Houzel 2016). The product that resulted is a domain-general cognitive architecture that strikes a homeostatic balance between metabolic efficiency and functional capacity.

By demonstrating the systemic functional adaptedness of the architecture undergirding central cognitive, this alternative to MM satisfies the conditions set by Cosmides and Tooby (1997) in the selection pressures argument. The force of the selection pressures argument relies principally on stable, recurrent adaptive problems inherent in ancestral environments. As demonstrated in the foregoing exposition of neural networks, the adaptive problems associated with informational distribution and metabolic efficiency correspond to stable, recurrent properties in the environment (albeit in the internal neural physiology of organisms embedded in the ancestral environment). These observations should motivate a paradigmatic shift in evolutionary psychology away from EP, along with its commitment to MM, and a move toward embracing an evolutionary logic that accounts for the domain-general properties of central cognition.

\section{Defenses of Massive Modularity}

Partisans of EP and the MM thesis would challenge the inferences drawn from neural network models in support of domain-general properties of cognitive architecture. There are at 
least two types of rebuttal that could reinforce the selection pressures argument established by Cosmides and Tooby. First, one may argue that the rich club and hub core structures should be regarded as modular systems, evidence that would militate in favor of the MM thesis. This approach calls for a demonstration that a cognitive module could perform the role of mitigating metabolic cost while functionally integrating neural network hubs.

This defense of the MM thesis hinges on whether the rich club and hub core structures satisfy the conditions for modularity. More explicitly, both network structures should be domainspecific, encapsulated, and mandatory in operation. Demonstrating that both structures act as a control system or switchyard of sorts would reinforce such an argument. Roughly speaking, control systems and switchyards are information-exchange channels that traffic information to disparate interconnected network modules. However, the processing of these inputs and the computations in central cognition would take place in specialized modules, ${ }^{8}$ rather than in the rich club or hub core. Such a limited functional role would accord straightforwardly with the three conditions of modularity.

The MM theorist is committed to regarding the rich club and hub core as modules. If functionally defined as switchyards, then the domain-specific functions of these network structures would relate to the retrieval and transmission of information between neural communities. This switchyard module would contribute metabolic efficiency by shortening pathways of inter-connection among the network hubs with which it interfaces. The switchyard would be encapsulated insofar as its circumscribed database is strictly limited to signals triggering distribution of information. Because a switchyard does not process the content of

\footnotetext{
${ }^{8}$ Carruthers (2006), for instance, proposes that working memory or the global workspace satisfies this role. On this account, neither of these mechanisms perform cognitive operations, but rather relay information to modules. On Sperber's account $(1994,2000)$ the metarepresentational module correspond to higher-order modules that traffics information among modular systems.
} 
signals, it does not access a wide range of cognitive databases to carry out its function. The switchyard would also respond to input and output signals mandatorily. Accordingly, a switchyard responds automatically upon receiving inputs and promptly sends the information to its appropriate sub-systems further downstream in central cognition. Because a switchyard satisfies the three modularity conditions, there is no need to posit a non-modular or domaingeneral system. Thus, comporting with the MM thesis, central cognition would be assembled by domain-specific modules.

The second defense of EP and the MM thesis offers a more detailed evolutionary account of central cognition by appealing to isolable adaptive problems found in the Environment of Evolutionary Adaptedness. Proponents of MM point to a circumscribed set of selection pressures leading to the development of central cognition. One such proposal is provided by the social exchange theory of reasoning, also called the "social contract theory" (Tooby and Cosmides 1985, 1989; Gigerenzer and Hug 1992). The social exchange theory posits selection pressures that particularly relate to the emergence of central cognition. Cosmides and Tooby (1992) assert that calculations of perceived costs and benefits prompted by instances of cooperation, resourceexchange, competition, and other socially relevant adaptive problems collectively shaped the mind to develop operations of central cognition.

To provide an example of how socially relevant selection pressures could select for features of central cognition, Gigerenzer and Hug (1992) point to the pragmatics of resource exchange. This adaptive problem would have selected for domain-specific modules that confront the judgment and decision-making demands imposed by negotiations over resource acquisition. In order for $x$ to decide whether to cooperate with $y$, a cascade of hierarchically arranged modular activations underlies the computations involved in such judgments (Carruthers 2006; Sperber 
2004). Modules further downstream in central cognition generate a decision whether to cooperate and later reciprocate with $y$ or otherwise to decline cooperation with $y$ in the resource exchange. The ultimate decision culminates from a series of algorithms relating to cost-benefit analyses, rule-following, and so on. This massively modular schema presupposes an assembly of modules that perform the requisite computations. For example, Boyer (2015) posits a module pertaining to OWNERSHIP as one such sub-component undergirding social reasoning: “The complex of intuitions generally called ownership are the outcome of largely tacit computations concerning the relative costs and benefits of using, guarding, or poaching resources, as well as collaborating with others in these diverse courses of action" (pp. 190). Likewise, adaptive problems related to cheater-detection, mate selection, etc., would exert selection pressures that account for the remaining functional properties of central cognition. This methodology forms the basis for a massively modular cognitive architecture functionally defined by the properties of social exchange.

\section{The Evolution of Domain-General Central Cognition}

In this section, I respond to the two foregoing defenses in defense of EP and the MM thesis. Against the first, I argue that the functional properties of rich club and hub core structures exceed those that define cognitive modules. Against the second defense, I argue that the social exchange theory only invites further objections that are otherwise satisfied by a domain-general cognitive architecture.

To start, I show how a topologically central placement situates the rich club and hub core as central control systems in cognition. The objective is to reinforce the argument that the rich club and hub core carry out systemic functions - that is, balancing metabolic cost and functional integration - by acting as an integrative hub in central cognition. Such a structure would fail to 
meet the three criteria of modularity - i.e., it would not perform functions characterized as (a) domain-specific, (b) encapsulated, or (c) operationally mandatory. The following strands of evidence drawn from neuroimaging studies demonstrates how the domain-general properties of the rich club and hub core enable non-modular systems to play an essential role in central cognition.

The rich club and hub core are network structures strongly correlated with the evolutionarily more recent central cognitive operations (Senden et al. 2017). However, the appearance of novel functions like analogical reasoning need not imply the emergence of a specialized sub-system arising to perform that function. As observed by Anderson and Penner-Wilger (2013), "the later something emerges, the more potentially useful existing circuitry there will be" (44). The repurposing of pre-existing neural circuitry for a wider range of novel functions is referred to as "neural reuse" (Anderson and Penner-Wilger 2013). The most plausible candidates for neural reuse as supporting structures in central cognition are those centrally-placed network structures optimally positioned to integrate a diverse range of neural processing areas (Senden et al. 2014). Fodor anticipated the discovery of such mechanisms in The Modularity of Mind:

Input analyzers, with their [...] relatively rigid domain specificity and automaticity of functioning, are the aboriginal prototypes of inference-making psychological systems. Cognitive evolution would thus have been in the direction of gradually freeing certain sorts of problem-solving systems from the constraints under which input analyzers labor - hence of producing, as a relatively late achievement, the comparatively domain-free inferential capacities which apparently mediate the higher flights of cognition. (1983: 43, emphasis added) 
Although Fodor would later disavow Darwinian research programs carried out in evolutionary psychology (see Fodor and Piattelli-Palmarini [2010]), this observation presages the hypothesis that central cognition emerged by structuring pathways of interconnectivity among specialized processors that were formerly segregated both anatomically and functionally. Fodor's reference to the "constraints" under which sub-system labor is just the sort of causal process that could give rise to central cognition. Similarly, Mithen (1996) described the evolutionary event leading to central cognition as a semi-breakdown in strict segregation among isolable cognitive systems.

On this proposal, the rich club and hub core are structures that break down functional constraints under which specialized sub-systems operate, thereby facilitating the complex computations of central cognition. The role originally posited for the rich club and hub core is structural integration, thereby shortening pathways of communication among interconnected subsystems (Sporns and Bullmore 2010; 2012: 337; van den Heuvel et al. 2012: 11372; Baggio et al. 2015). Structural integration contributes metabolic efficiency and sets background conditions for the development of functions that integrate outputs from different sub-systems (Cocchi et al. 2014). In order to implement central cognition, "there must be relatively nondenominational (i.e., domain-inspecific) psychological systems which operate, inter alia, to exploit the information that input systems provide" (Fodor 1983: 103). Functional integration accounts for the capacity to combine contents from a range of semantic databases into complex representations (Fodor 1994; Fodor and Lepore 1996).

According to the present framework, functional integration may be understood as an exaptation built upon structural integration. An exaptation refers to the assignment of novel functions to pre-existing biological structures. By analogy, feathers originally evolved for thermal regulation, which were reassigned to flight capacities or to signaling among conspecifics 
(Persons and Currie 2015). Feathers confer compounded adaptive benefit by overlaying a multitude of distinct functions. It is not uncommon to observe mechanisms take on functions that diverge from older, etiological functions (Sterelny and Griffiths 1999: 320). Likewise, some cognitive mechanisms followed a pattern of cumulatively "jury-rigging" additional functions relating to central cognition on pre-established structural pathways.

Schulz (2008) observes that some traits evolve in tandem with others as complex traits as a result of compounding fitness value. Accordingly, the adaptive value of traits encoding for structural connectivity compound considerably when causally linked to traits supporting functional connectivity. Fig. 6 below illustrates the significant overlap in structural and functional connections among various resting states networks (RSNs), following the hypothesis that both forms of connectivity are strongly linked to one another as complimentary network properties.

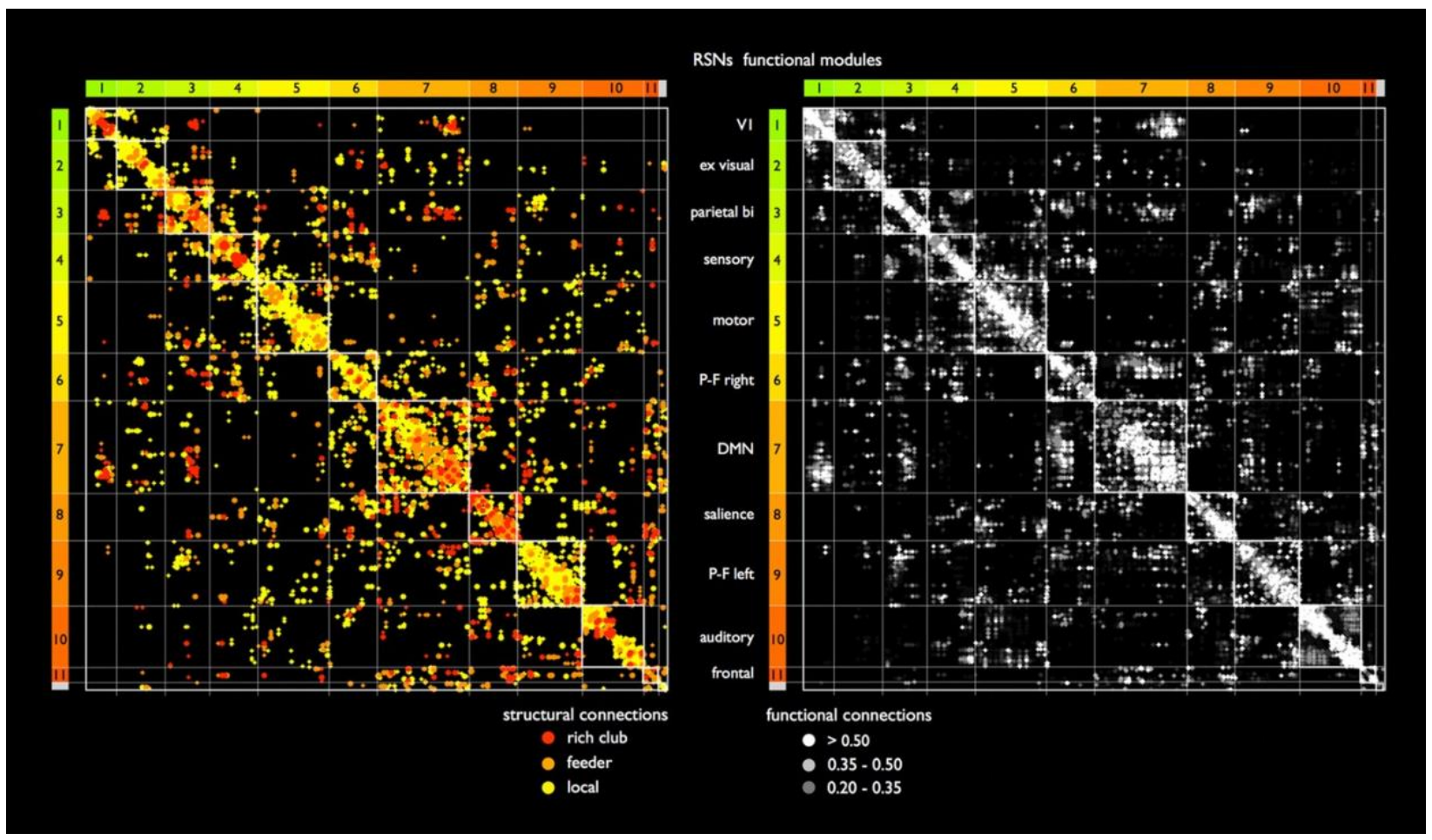




\section{Fig. 6: Structural and Functional Connectivity Matrices}

Analysis of 11 resting state networks (RSNs) reveals the complimentary development of structural connections and functional connections among discrete processing areas. Correlations along the dimension of structural connectivity are denoted as local (within a neural community), feeder (between hubs connecting neural communities), or rich club (referring to the most globally integrated network connections). The other dimension of functional connectivity designates the strength of functional coupling among distinct RSNs. Reprinted with permission from van den Heuvel and Sporns (2013: 14497).

Structural connections enable the development of functional connections, where compounding fitness results from these complimentary properties. Supposing that structural integration and functional integration can diverge from one another along separate trajectories of evolutionary development, overdevelopment of either trait without regard for other factors would eventually incur fitness costs. Unmoderated development of functional capacities would impose metabolic costs and diminish network efficiency. Conversely, maximizing efficiency by restricting the number of functional connections would limit functional capacity and curtail cognitive flexibility. On the other hand, if regarded as complimentary traits, the same evolutionary trajectory leading to the distribution of information flow in neural sub-systems also guided the development of dynamical processes that integrate representations in central cognition, thereby striking a balance between the two demands.

There is a growing body of evidence from neuroimaging and network models suggesting that the rich club and hub core structures actively participate in the structural and functional integration of information in central cognition (see Zamora-Lòpez et al. 2009; Bullmore and Sporns 2012; van den Heuvel et al. 2012). These models suggest that the rich club and hub core carry out functions that exceed those of domain-specific cognitive modules.

The following observations establish the domain-general properties of the rich club and hub core. The cortical regions corresponding to these network structures have a distinctively high 
'participation index,' an indicator of participation across a wide range of cognitive tasks associated with global processing of information (Bullmore and Sporns 2012: 342). Anderson and Pessoa (2011) performed a task-diversity analysis, which measures the range of cognitive tasks pertaining to a neural system, revealing cortical correlates of the rich club and hub core that support a multitude of cognitive constructs, including the allocation of attention, retrieval of information from semantic memory, and buffering contents in working memory. They also measured the functional diversity of 78 different cortical regions from 0 to 1 (i.e., the closer to 1 , the more diverse the functional role of that cortical region). They determined that the average diversity of these cortical regions was .70 , averaged over 1,138 experimental tasks along 11 different BrainMap task domains. These BrainMap items relate to cognitive domains that include semantic memory, reasoning, language semantics and working memory (for elaboration on BrainMap domains, see Fox et al. [2005]). Applying network analysis to functional magnetic resonance imaging (fMRI) data, Shine et al. (2016) detected activation in these cortical areas during performance on cognitive tasks that measure higher-order constructs such as relational reasoning. An investigation into dynamical properties of neural networks uncovered a negative correlation between clustered, modular processing and cognitive effort - especially in working memory tasks associated with central cognition - and positive correlation with more globally integrated configuration of processing (Kitzbichler et al. 2011: 8259). Uttal (2001) found through fMRI that vast integrated neural networks facilitate complex reasoning tasks, rather than heterogeneous, specialized sub-systems. Yue et al. (2017) and Cohen and D’Esposito (2016) discovered that static modular organization and central cognitive task activation are negatively correlated, with rapid reconfiguration of integrative networks scaling up commensurately with increasing task complexity. Further analysis of dynamic network changes during cognitive 
control and reasoning tasks failed to identify any isolable sub-system that carries out central cognition (Cocchi et al. 2013; Cole et al. 2013; Bola and Sabel 2015).

These findings also suggest that the neural correlates of the rich club and hub core are unencapsulated with respect to accessible semantic databases. Van den Heuvel (2012) measured communication pathways of sub-systems in the connectome and determined that at least $69 \%$ of communication pathways pass through these centralized structures, indicating access to a broad range of informational domains. A prior study by Scannell et al. (1995) revealed that the rich club and hub core functionally integrate information across cortical networks ranging from the fronto-limbic, visual, auditory, to somatosensory and motor processing regions.

Finally, the rich club and hub core also appears to be flexible controllers in central cognitive tasks. The corresponding cortical regions have been described appropriately as a collection of "multi-demand systems" (Fedorenko 2014: 4). The multi-demand systems have been shown to support task-setting and task-switching roles in "attention (Posner and Petersen 1990; Desimone and Duncan 1995; Peterson and Posner 2012), working memory (Goldman-Rakic 1995), cognitive control (Miller and Cohen 2001; Koechlin et al. 2003; Badre and D’Esposito 2009), structure building/unification (Hagoort 2005), timing and/or sequencing (Luria 1966; Janata and Grafton 2003; Fuster 2008), attentional episodes in goal-directed behavior (Duncan 2010), and conscious awareness (Dehaene and Changeux 2011)" (cf. Fedorenko 2014: 4). ${ }^{9}$ These findings reinforce the ascription of flexibility to the rich club and hub core structures, properties that are inconsistent with the defining properties of modularity.

\footnotetext{
${ }^{9}$ Connecting the present analysis of central cognition with theories of consciousness, it would be worth exploring further whether the cortical regions undergirded by rich club/ hub cores also instantiate a global neuronal workspace (see Baars 1988; 1997; 2002). Following up on this question is beyond the purview of the present discussion, but further investigation may prove worthwhile.
} 
Having established the extensive functional repertoire of the rich club cognitive architecture, we now turn to address the social exchange theory of central cognition. The social exchange hypothesis runs afoul of the so-called "grain problem" (Sterelny and Griffiths 1999; Atkinson and Wheeler 2003). Deciding on the appropriate level or "grain" of description in identifying the selected function of a cognitive mechanism is often arbitrary and erroneously atomistic. Any single adaptive problem may be analyzed into an array of separate adaptive problems, where each imposes a selection pressure favoring a corresponding cognitive module that varies only with the level of description. To take Boyer's (2015) example of OWNERSHIP, is this property of social exchange a single adaptive problem or rather a complex set of multiple distinct problems (Sterelny and Griffiths 1999: 328)? The problem of OWNERSHIP may be analyzed into separate components relating to the perceptual cues of property, and group affiliation, and cost-andbenefit analysis. Each re-description alters the adaptive problem selecting for the cognitive module along with its proper domain, thus the encapsulated database of the corresponding module would be overdetermined as either the perceptual cues of property, or group affiliation, or cost-benefit calculation. The alternative of delineating the proper domain of OWNERSHIP as a hierarchical assembly of modules does not resolve the grain problem either. The particular ordering of the hierarchy would be arbitrary, for it may turn out that the orthogonal arrangement of computations proceeds from perceptual cues of property, to group affiliation, to cost-andbenefit analysis - or in the exact reverse order. And, finally, the remaining option of grouping these distinct domains together under the single rubric of OWNERSHIP violates the informational encapsulation criterion of modularity. Because the proper domain of the OWNERSHIP module would encompass the databases of each component, by implication the highest-order module would actually be unencapsulated and, insofar as it requires access to the databases of each 
subordinate module, it would also be domain-general in its capacity to perform formal algorithms that operate on a variety of decision-rule structures. Thus, when facing the grain problem, the MM cognitive architecture threatens to collapse into a variant of the domaingeneral central systems theory.

Indeed, it is misleading to define the etiology of a cognitive mechanism by reference to any single environmental influence. Recalling Symons (1992) observation that there is no general adaptive problem to be found in the ancestral environment, it would be just as accurate to state there is no isolated adaptive problem. As is the case with OWNERSHIP, the description of any adaptive problem countenances innumerable re-descriptions that reflect a tangled web of interrelated adaptive problems.

The domain-general cognitive architecture proposed here avoids the grain problem altogether by remaining non-committal and flexible on questions of the proper level of description in denoting environmental demands. There is no obligation to make a committed stance on how social exchange, ownership, relative status, or cost-benefit analysis are in fact related to one another conceptually. The proposed alternative to the MM thesis nevertheless allows for properties of social exchange to play a complimentary role in shaping the sorts of contents available in central cognition without necessarily defining the functionality of the central cognitive mechanisms. A cognitive architecture organized around the principle of systemic functional adaptedness orients the proper level of analysis toward the containing neural system rather than appealing to features of the external environment. Instead of endeavoring to decipher a proper grain of analysis, evolutionary psychology should affirm cognitive architectures that accurately reflect the demonstrable inter-relatedness of adaptive problems and the highly variable ecology faced by hominid ancestors. Hence the hypothesis that cognitive mechanisms 
were selected on the basis of causal contributions to structural and functional integration, allowing for the flexible performance of computations applicable to numerous models of the ancestral environment. Far from demonstrating untenability of domain-general cognition, the theoretical underpinnings of evolutionary psychology underscore the relative fitness advantages on offer by domain-general central systems.

Having responded to both defenses of EP and the MM thesis, it is important to flag limitations and directions for further developing the proposed framework. One potentially tendentious assumption is that structural and functional integration evolved as complimentary traits due to fitness advantages these variants would enjoy over competitors. However, the mechanisms that evolve are not always the most optimal conceivable solutions to adaptive problems (Barrett 2015: 78). Our cognitive architecture may be suboptimal in the space of all conceivable variants, but good enough to impart fitness advantages that propagated the genes of hunter-gatherer ancestors. Another limitation of the proposed framework is its lacking a method for quantifying the relation between expanses in cortical volume and corresponding investments in the rich club and hub core structures during evolutionary development. ${ }^{10}$ The imperative to establish a suitable algorithm or set of equations for the task becomes clear when considering cross-species comparisons of neural network properties. For instance, analogous structural and functional characteristics of the rich club and hub core have been identified in network models of the cat cortex (Zamora-Lòpez et al. 2009, 2011) and macaque cortex (Harriger et al. 2012), not just the human cortex (van den Heuvel and Sporns 2011). If the rich club and hub core support properties of central cognition, then there should be evidence of central cognition in proportion

\footnotetext{
${ }^{10}$ What needs to be determined is whether this commensurate scaling up of neural integration and cognitive complexity is a linear or non-linear relation. While I do not address such concerns here, these details could be ascertained through further investigation and development of the proposed framework.
} 
to the development of these neural network structures (measured in terms of volume and density of structural connectivity among neural sub-systems). One basis for evaluating stated claims about the rich club and hub core is obtaining measurements of the relative degrees of central cognitive functions exhibited by organisms equipped with similar network configurations. A third limitation is that the methodology of mapping neural network components onto properties of cognitive architecture indulges in speculation to some degree (Weiskopf 2016). The current state of network science and connectomics allow for divergent interpretations of the underlying cognitive architecture. More research must be conducted to discover the representational format in which the rich club and hub core carry out cognitive functions. More precisely, current findings allow for (but do not necessarily entail) the ascription of generally applicable logical rules and formal algorithms to the cognitive operations performed by these network components.

\section{Conclusion}

Despite these worries and limitations, the evidence adduced in this paper casts sufficient doubt on the prospect of inferring the MM thesis from evolutionary psychology. The absence of isolable adaptive problems that account for the evolution of central cognition should motivate consideration of alternative methodologies. A viable alternative would reject the assignment of functional roles to cognitive mechanisms by appealing to properties of the external environment. By reformulating adaptive functions in terms of their causal contribution to internal cognitive architecture, evolutionary psychologists may posit a domain-general cognitive architecture that offers not only a broadened explanatory scope, but also averts objections that beset massively modular architectures. Further empirical investigation across the cognitive sciences are still 
required, but the currently available evidence points to a plausible evolutionary account of the selection pressures that gave rise to domain-general central cognition. 


\section{References}

Amundson R, Lauder G (1994) Function without purpose: The uses of causal role function in evolutionary biology. Biology \& Philosophy 9(4): 443-469

Anderson ML, Penner-Wilger M (2013) Neural Reuse in the Evolution and Development of the Brain: Evidence for Developmental Homology? Developmental Psychobiology 55(1): $42-51$

Anderson ML, Pessoa L (2011) Quantifying the diversity of neural activations in individual brain regions. In: Carlson L, Hölscher C, Shipley T (eds), Proceedings of the $33^{\text {rd }}$ Annual Conference of the Cognitive Science Society, Austin, TX: Cognitive Science Society, pp $2421-2426$

Atkinson A, Wheeler M (2003) Evolutionary psychology's grain problem and the cognitive neuroscience of reasoning. In: Over D (ed), Evolution and the psychology of reasoning: The debate, Hove: Psychology Press, pp 61-99

Baars B (2002) The conscious access hypothesis: Origins and recent evidence. Trends in Cog. Sci. 6 (1): 47-52

Baars B (1997) In the Theater of Conciousness. New York, NY: Oxford University Press

Baars B (1998) A Cognitive Theory of Consciousness. Cambridge, MA: Cambridge University Press

Badre D, D'Esposito M (2009) Is the rostro-caudal axis of the frontal lobe hierarchical? Nat. Rev. Neurosci.10: 659-669 
Baggio HC, Segura B, Junque C, de Reus MA, Sala-Llonch R, van den Heuvel MP (2015) Rich Club Organization and Cognitive Performance in Healthy Older Participants. $J$ Cogn Neurosci 27(9): 1801-1810

Barrett CH (2015) The Shape of Thought: How Mental Adaptations Evolve. New York, NY: Oxford University Press

Barrett CH (2012) A Hierarchical Model of the Evolution of Human Brain Specialization. PNAS 109(1): 10733-10740

Bola M, Sabel BA (2015) Dynamic reorganization of brain functional networks during cognition. NeuroImage 144: 398-413

Boureau Y, Sokol-Hessner P, Daw ND (2015) Deciding How to Decide: Self-Control and meta Decision Making. Trends in Cognitive Sciences 19(11): 700-710

Boyer P (2015) How Natural Selection Shapes Conceptual Structure. In: Margolis E, Lawrence S (eds), The Conceptual Mind: New Directions in the Study of Concepts, Cambridge: MIT Press, pp 185-200

Brewer B (1999) Perception and Reason. Oxford: Oxford University Press

Buller D (2005) Adapting Minds: Evolutionary Psychology and the Persistent Quest for Human Nature, MA: MIT Press

Bullmore E, Sporns O (2009) Complex brain networks: graph theoretical analysis of structural and functional systems. Nature Review of Neuroscience 10: 186-198

Bullmore E, Sporns O (2012) The Economy of Brain Network Organization. Nature Review Neuroscience 13(5): 336-349 
Bunge SA, Wendelken C, Badre D, Wagner AD (2005) Analogical reasoning and prefrontal cortex: Evidence for separable retrieval and integration mechanisms. Cerebral Cortex 15(3): 239-249

Buss D (2005) The Handbook of Evolutionary Psychology, NJ: John Wiley and Sons Buss D (1995) Evolutionary Psychology: A New Paradigm for Psychological Science. Psychological Enquiry 6(1): 1-30

Caramazza A, Shelton J (1998) Domain-specific knowledge systems in the brain: The animate inanimate distinction. Journal of Cognitive Neuroscience 10: 1-34

Carruthers P (2013a). On Central Cognition. Philosophical Studies 170(1): 143-162

Carruthers P (2013b) Evolution of Working Memory. PNAS 110(2): 10371-10378

Carruthers P (2006) The Case for Massively Modular Models of Mind. In: Stainton RJ (ed), Contemporary Debates in Cognitive Science, New Jersey: Wiley-Blackwell, pp 3-21

Carruthers P (2004) The Mind is a System of Modules Shaped by Natural Selection. In: Hitchcock C (ed), Contemporary Debates in Philosophy of Science. New Jersey: Wiley Blackwell, pp 293-311

Chklovskii DB, Koulakov AA (2004) Maps in the brain: what can we learn from them? Anпu. Rev. Neurosci. 27: 369-392

Cocchi L, Zalesky A, Fornito A, Mattingley JB (2013) Dynamic cooperation and competition between brain systems during cognitive control. Trends in Cog. Sci. 17(10): 493-501

Cohen JR, D’Esposito M (2016) The Segregation and Integration of Distinct Brain Networks and Their Relationship to Cognition. J. Neurosci. 36: 12083-12094

Cole MW, Reynolds JR, Power JD, Repovs G, Anticevic A, Braver TS (2013) Multi-task connectivity reveals flexible hubs for adaptive task control. Nat. Neurosci. 16: 1348-1355 
Cosmides L, Tooby J (2000) The Cognitive Neuroscience of Social Reasoning, In: Gazzaniga MS (ed), The New Cognitive Neurosciences, Second Edition. Cambridge, MA: MIT Press, pp 1259-1270

Cosmides L, Tooby J (1994) Origins of Domain Specificity: The Evolution of Functional Organization. In: Hirschfield LA, Gelman S (eds), Mapping the Mind, Cambridge: Cambridge University Press, pp 85-116

Cosmides L, Tooby J (1997) The Modular Nature of Human Intelligence. In: Scheibel A, Schopf JW (eds), The Origins and Evolution of Intelligence, MA: Jones and Bartlett Publishers, pp 71-101

Cummins R (1975) Functional Analysis. The Journal of Philosophy 72(20): 741-765

Davies PS (2000) The nature of natural norms: Why selected functions are systemic capacity functions. Nous 34(1): 85-107

Davies PS, Fetzer J, Foster T (1995) Logical reasoning and domain specificity - A critique of the social exchange theory of reasoning. Biology and Philosophy 10 (1): 1-37

Dehaene S, Changeux JP (2011) Experimental and theoretical approaches to conscious processing. Neuron 70: 200-227

Desimone R, Duncan J (1995) Neural mechanisms of selective attention. Annu. Rev. Neurosci. 18: 193-222

De Reus MA, van den Heuvel MP (2014) Simulated rich club lesioning in brain networks: a scaffold for communication and integration? Front Hum Neurosci 8 (647): 1-5

De Reus MA, van den Heuvel MP (2013). Rich Club Organization and Intermodule Communication in the Cat Connectome. The Journal of Neuroscience 33(32): 12929 12939 
Duncan J (2010) The multiple-demand (MD) system of the primate brain: mental programs for intelligent behaviour. Trends Cogn. Sci. 14: 172-179

Elman JL, Bates, EA, Johnson MH, Karmiloff-Smith A, Parisi D, Plunkett K (1996)

Rethinking Innateness: A Connectionist Perspective on Development. Cambridge, MA: MIT Press

Evans G (1982) The Varieties of Reference. Oxford: Oxford University Press

Fedorenko E (2014) The role of domain-general cognitive control in language comprehension. Front. Neurosci. 5 (335): 1-17

Fodor J, Lepore E (1996) The red herring and the pet fish: why concepts still can't be prototypes. Cognition 58: 253-270

Fodor J, Piattelli-Palmarini M (2010) What Darwin Got Wrong. New York: Farrar, Straus, Giroux

Fodor J, Pylyshyn Z (2015) Minds Without Meanings: An Essay On the Content of Concepts. MA: MIT Press

Fodor J (2000) The Mind Doesn't Work That Way. MA: MIT Press

Fodor J (1994) Concepts: A Potboiler. Cognition 50: 95-113

Fodor J (1983) The Modularity of Mind. MA: MIT Press

Fox PT, Laird A.R., Fox SP, Fox M, Uecker AM, Crank M, Lancaster JL (2005) BrainMap taxonomy of experimental design: Description and evaluation. Human Brain Mapping 25: 185-198

Fuster J (2008) The Prefrontal Cortex, Fourth Edition. London: Academic Press

Gigerenzer G, Hug K (1992) Domain-specific reasoning: Social contracts, cheating, and perspective change. Cognition 43: 127-171 
Godfrey-Smith P (2013) Philosophy of Biology, NJ: Princeton University Press

Godfrey-Smith P (2010) It Got Eaten. London Review of Books 32 (13): 29-30

Godfrey-Smith P (1986) Complexity and the Function of Mind in Nature. Cambridge, MA:

Cambridge University Press

Goldman-Rakic PS (1995) Cellular basis of working memory. Neuron 14: 477-485

Gould SJ, Vrba ES (1982) Exaptation - A Missing Term in the Science of Form. Paleobiology $8(1): 4-15$

Grayson DS, Ray S, Carpenter S, Iyer S, Costa Dias TG, Stevens C, Nigg JT, Fair DA (2014)

Structural and Functional Rich Club Organization of the Brain in Children and Adults. PLOS ONE 9 (2): e88297

Green M (2016) Expressing, Showing, and Representing. In: Abel C, Smith J (eds) Emotional Expression: Philosophical, Psychological, and Legal Perspectives. New York: Cambridge University Press, pp 1-24.

Hagoort P(2005) On Broca, brain and binding: a new framework. Trends Cogn. Sci. 9: 416-423

Harman G (1986) Change in view: Principles of Reasoning. Cambridge, MA: MIT Press.

Harriger L, van den Heuvel MP, Sporns O (2012) Rich club organization of macaque cerebral cortex and its role in network communication. PLoS 7: e46497

Herculano-Houzel S (2016) The Human Advantage: A New Understanding of How Our Brain Became Remarkable, MA: MIT Press

Holyoak K (2012) Analogy and Relational Reasoning. In: Holyoak KJ, and Morrison RG (eds), The Oxford handbook of thinking and reasoning, New York: Oxford University Press, pp $234-259$ 
Hurley S (2006) Making sense of animals. In: Hurley S, Nudds M (eds), Rational Animals? Oxford: Oxford University Press

Janata P, Grafton, ST (2003) Swinging in the brain: shared neural substrates for behaviors related to sequencing in music. Nat. Neurosci. 6: 682-687

Jungé JA, Dennett DC (2010) Multi-use and constraints from original use. Behavioral and Brain Sciences, 33: 277-278

Kaiser M, Hilgetag CC (2006) Nonoptimal component placement, but short processing paths, due to long-distance projections in neural systems. PloS Comp. Biol. 2: e95

Karmiloff-Smith A (1992) Beyond Modularity: A Developmental Perspective on Cognitive Science. Cambridge, MA: MIT Press

Kitzbichler MG, Henson RNA, Smith ML, Nathan PJ, Bullmore ET (2011) Cognitive Effort Drives Workspace Configuration of Human Brain Functional Networks. J. Neurosci. 31(22): $8259-8270$

Koechlin E, Ody C, Kouneiher F (2003) The architecture of cognitive control in the human prefrontal cortex. Science 302: 1181-1185

Krawczyk D (2018) Reasoning: The neuroscience of how we think. Cambridge, MA: Elsevier Krawczyk D (2010) The cognition and neuroscience of relational reasoning. Brain Research 1428: $13-23$

Liang X, Hsu LM, Lu H, Sumiyoshi A, He Y, Yang Y (2017) The Rich-Club Organization in Rat Functional Brain Network to Balance Between Communication Cost and Efficiency. Cereb. Cortex: 1-12

Luria AR (1966) Higher Cortical Functions in Man. B. Haigh (trans.) New York, NY: Basic Books 
Michel M (2017) A role for the anterior insular cortex in the global neuronal workspace model of consciousness. Consciousness and Cognition 49: 333-346

Miller EK, Cohen JD (2001) An integrative theory of prefrontal cortex function. Annu. Rev. Neurosci. 24: 167-202

Mithen S (1996) The Prehistory of the Mind, London: Thames and Hudson Ltd

Nettle D (2007) A Module for Metaphor? The Site of Imagination in the Architecture of the Mind. Proceedings of the British Academy 147: 259-274

Petersen SE, Posner MI (2012) The attention system of the human brain: 20 years after. Annu. Rev. Neurosci. 35: 73-89.

Persons WS, Currie PJ (2015) Bristles before down: a new perspective on the functional origin of feathers. Evolution 69: 857-862

Pinker S (1997) How the Mind Works, New York: Norton

Pinker S (1994) The Language Instinct, New York: William Morrow \& Co

Posner MI, Petersen SE (1990) The attention system of the human brain. Annu. Rev.

Neurosci. 13: 25-42

Prinz J (2006) Is the Mind Really Modular? In: Stainton RJ (ed), Contemporary Debates in Cognitive Science, New Jersey: Wiley-Blackwell, pp 22-36

Quartz SR, Sejnowski TJ (1997) The Neural Basis of Cognitive Development: A Constructivist Manifesto. Behavioral and Brain Sciences. 20(4): 537-556

Uddin LQ (2015) Salience processing and insular cortical function and dysfunction. Nature Reviews Neuroscience 16(1): 55-61 
Unsworth N, Robison MK (2017) A locus coeruleus-norepinephrine account of individual differences in working memory capacity and attention control. Psychonomic Bulletin \& Review 24(4): 1282-1311

Rosch E (1978) Principles of Categorization. In: Roach E, Lloyd B (eds), Cognition and Categorization, New Jersey: Lawrence Erlbaum Associates, 27-48

Samuels R (1998) Evolutionary Psychology and the Massive Modularity Hypothesis. Brit J. Phil. Sci. 49: 575-602

Scannell JW, Blakemore C, \& Young MP (1995) Analysis of connectivity in the cat cerebral cortex. J Neurosci 15: 1463-1483

Schulz, A (2008) Structural flaws: massive modularity and the argument form design. Brit. J. Phil. Sci. 59: 733-743

Senden M, Reuter N, van den Heuvel MP, Goebel R, Deco G (2017) Cortical rich club regions can organize state-dependent functional network formation by engaging in oscillatory behavior. NeuroImage 146: 561-574

Senden M, Deco G, de Reus MA, Goebel R, van den Heuvel MP (2014) Rich club organization supports a diverse set of functional network configurations. NeuroImage 96: 174-182

Shine JM, Bissett PG, Bell PT, Oluwasanmi K, Balsters JH, Gorgolewski KJ, Moodie CA, Poldrack RA (2016) The Dynamics of Functional Brain Networks: Integrated Network States during Cognitive Task Performance. Neuron 92: 1-11

Sober E (1984) The Nature of Selection: Evolutionary Theory in Philosophical Focus, Chicago: The University of Chicago Press 
Sperber D (2004) Modularity and Relevance: How Can a Massively Modular Mind Be

Flexible and Context-Sensitive? In: Carruthers P, Laurence S, Stich S (eds), The Innate Mind: Structure and Content, New York: Oxford University Press, pp 53-68

Sperber D (2002) In Defense of Massive Modularity. In: Dupoux E (ed), Language, Brain, and Cognitive Development, MA: MIT Press, pp 47-57

Sperber D (2000) Metarepresentations in an Evolutionary Perspective. In: Sperber D (ed), Metarepresentations, Oxford: Oxford University Press, pp 117-137

Sperber D (1994) The Modularity of Thought and the Epidemiology of Representations. In: Hischfield LA, Gelman SA (eds), Mapping the Mind, Cambridge: Cambridge University Press, pp 39-67

Sporns O (2013) Network attributes for segregation and integration in the human brain. Curr. Opin. Neurobiol. 23: 162-171

Sporns O (2012) Discovering the Human Connectome. MA: MIT Press Sporns O (2010) Networks of the Brain. MA: MIT Press

Sterelny K, Griffiths PE (1999) Sex and Death: An Introduction to Philosophy of Biology. Chicago: The University of Chicago Press

Symons D (1992) On the Use and Misuse of Darwinism in the Study of Human Behavior. In: Barkow JH, Cosmides L, Tooby J (eds), The Adapted Mind: Evolutionary Psychology and the Generation of Culture, New York: Oxford University Press, pp 137-159

Tooby J, Cosmides L (2000) Toward Mapping the Evolved Functional Organization of Mind and Brain, In: Gazzaniga MS (ed), The New Cognitive Neurosciences, Second Edition. Cambridge, MA: MIT Press, pp 1167-1178 
Tooby J, Cosmides L (1995) Foreword in Baron-Cohen S, Mindblindness: An Essay on Autism and Theory of Mind, Cambridge: MIT Press, pp xi-xviii

Tooby J, Cosmides L (1992) The Psychological Foundations of Culture. In: Barkow JH, Cosmides L, Tooby J (eds), The Adapted Mind: Evolutionary Psychology and the Generation of Culture, New York: Oxford University Press, pp 19-136.

Tooby J, Cosmides L (1987). Conceptual Foundations of Evolutionary Psychology. In: Buss DM (ed), Handbook of Evolutionary Psychology, Hoboken, NJ: John Wiley \& Sons, Inc.

Uttal WR (2001) The new phrenology: The limits of localizing cognitive processes in the brain. Cambridge, MA: MIT Press

Van den Heuvel MP, Sporns O (2013) An anatomical substrate for integration among functional networks in human cortex. J Neurosci 33(36): 14489-14500

Van den Heuvel MP, Kahn R, Goñi J, Sporns O (2012) High-cost, high-capacity backbone for global brain communication. PNAS 109(28): 11372-11377

Van den Heuvel MP, Sporns O (2011) Rich-club organization of the human connectome. $J$ Neurosci 31: 15775-15786

Vincent JL, Kahn I, Snyder AZ, Raichle ME, Buckner RL (2008) Evidence for a frontoparietal control system revealed by intrinsic functional connectivity. J Neurophysiol 100(6): 3328-3342

Weiskopf, D (2016) Integrative Modeling and the Role of Neural Constraints. Philosophy of Science Archive, 83(5): 675-685

Weiskopf, D (2014) The Architecture of Higher Thought. In: M. Sprevak M, Kallestrup J (eds), New Waves in Philosophy of Mind, NY: Palgrave Macmillan, pp 242-261

Weiskopf, D (2010) Concepts and the modularity of thought. Dialectica 64(1): 107-130. 
Woodward J, Cowie F (2004). The Mind is not (just) a System of Modules Shaped (just) by Natural Selection. In: Hitchcock C (ed), Contemporary Debates in Philosophy of Science. New Jersey: Wiley-Blackwell, pp 312-334

Yue Q, Martin R, Fischer-Baum S, Ramos Nuńez A, Ye F, Deem M (2017) Brain modularity mediates the relation between task complexity and performance. Journal of Cognitive Neuroscience, 9: 1532-1546

Zamora-Lòpez G, Zhou C, Kurths J (2009) Graph analyses of cortical networks reveals complex anatomical communication substrate. Chaos 19(1): 015117

Zamora-Lòpez G, Zhou C, Kurths J (2011) Exploring brain function from anatomical connectivity. Front. Neurosci. 5(83): 1-11

Zeman A, Obst O, Brooks KR, Rich AN (2013) The Müller-Lyer Illusion in a computational Model of Biological Object Recognition. PLoS 8 (2): e56126

Zerilli, J (2017) Against the "system" module. Philosophical Psychology, 30(3): 235-250 
